\title{
Extended Near-Infrared Resonance Raman Investigations of an Organic Mixed-Valence System: Diazatetracyclodiene Radical Cation
}

\author{
Robert D. Williams and Joseph T. Hupp* \\ Department of Chemistry, Northwestern University, Evanston, Illinois 60208 \\ Michael T. Ramm and Stephen F. Nelsen \\ Department of Chemistry, University of Wisconsin, Madison, Wisconsin
}

Received: August 7, 1999

\begin{abstract}
Resonance Raman scattering studies in the extended near-infrared region show that six modes are coupled to the intramolecular charge-transfer transition in the mixed-valence radical cation diazatetracyclodiene. Spectral analysis based on time-dependent scattering theories shows that all six modes make substantial contributions to the vibrational reorganization energy. Measured Raman scattering cross sections were found to be considerably less than would be predicted by the conventional time-dependent scattering theories utilized here, evidently owing to complications arising from avoided surface crossings and anharmonic wave packet propagation. An examination of the classical and nonclassical kinetic effects of the coupled modes indicated that a considerable influence on the rate of intramolecular electron transfer is exerted by the modes collectively.
\end{abstract}

\section{Introduction}

Mixed valence compounds are those possessing two redox centers where the centers nominally exist in different formal charge states. ${ }^{1}$ If the centers are compositionally identical (apart from charge), then a degenerate electron exchange can occur, with the rate of exchange governed by vibrational and solvational Franck-Condon effects, together with electronic or nuclear dynamical effects. Alternatively, if the centers are very strongly interacting, in an electronic sense, then the available odd valence electron(s) are intrinsically delocalized, the redoxcenter charges are identical, and the oxidation states are best characterized as fractional. The most extensively studied mixedvalence compounds clearly have been dinuclear coordination compounds consisting of group VIII transition metals bridged by organic ligands. ${ }^{2}$ These compounds feature intervalence charge transfer (IVCT) absorption bands in the red to nearinfrared region of the spectrum, which are, at least in a number of valence localized cases, well described by Hush's theory of intervalence charge transfer. ${ }^{3}$ In addition to the abundance of transition metal and organometallic based mixed-valence systems, a few purely organic mixed valence compounds have been prepared and studied. ${ }^{1,4}$ For example, Nelsen and co-workers have extensively examined systems such as the bis-hydrazine (1). ${ }^{5}$ One-electron oxidation of $\mathbf{1}$ yields a radical cation that

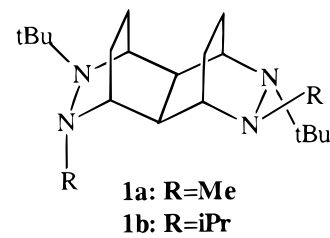

exhibits a transition in the visible region of the spectrum because of IVCT between the charge-bearing dinitrogen units. This transition has proven to be amenable to analysis via Hush's classical theory. ${ }^{3}$ Since electron transfer for $\mathbf{1}$ was found to be slow on the electron spin resonance (ESR) time scale, ${ }^{5}$ more recent work has focused on structural variants in which the electron-transfer rate is increased to be measurable by ESR line broadening. ${ }^{6-8}$

Related bis-hydrazyl dications $\left(\mathbf{2}^{2+}\right)$ consisting of two aza moieties separated by four $\sigma$ bonds have also been studied. ${ }^{5,9,10}$ As shown in eq 1, one-electron reduction of these leads to a

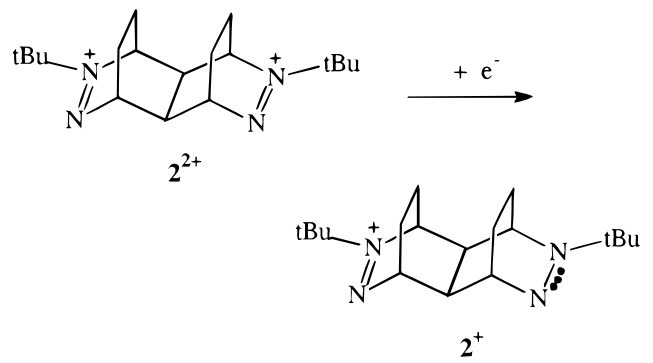

radical cation in which, again, charge is localized on a dinitrogen unit. The absorbance spectrum of the bis-hydrazyl species shown is characterized by a relatively broad, intense transition found in the near-infrared $\left(\lambda_{\max }=1070 \mathrm{~nm}\right)$, which has been assigned, as shown in eq 2, to IVCT. Recall that for symmetrical, valence-

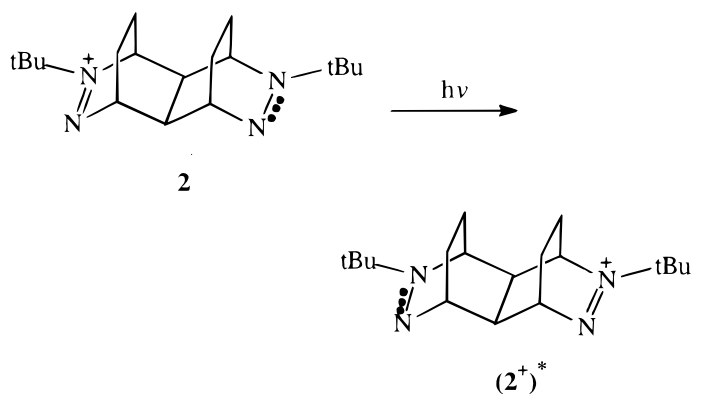

localized systems, the IVCT transition energy can be equated 
approximately to the total reorganization energy (vibrational + solvational reorganization energy) for electron transfer. The reorganization energy, in turn, can be related via Marcus-Hush theory to the classical activation energy for thermal electron transfer (cf. eq 1). For $2^{+}$, ESR measurements imply that the intramolecular electron-transfer rate (eq 3 ) exceeds $\sim 10^{8} \mathrm{~s}^{-1}$,

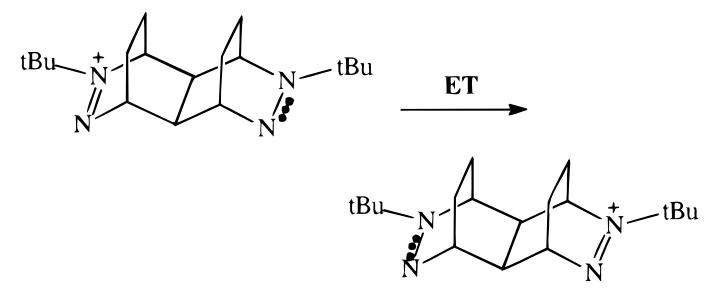

while the IVCT measurements imply that the hypothetical nonadiabatic ET barrier height (i.e., the classical activation free energy barrier height in the absence of electronic-couplinginduced barrier roundoff) is ca. $7 \mathrm{kcal} \mathrm{mol}^{-1}$.

Given the interest in both the kinetics and energetics of these unusual charge-transfer processes, we felt that it would be informative to characterize the reorganizational barrier in a more detailed way. The approach we chose was resonance enhanced Raman spectroscopy in the extended near-infrared. This technique has been profitably employed previously to generate fully mode-specific descriptions of the vibrational portion of the reorganization energy for electron transfer in inorganic mixedvalence systems. ${ }^{11}$ Here, we report the Raman scattering of the bis-hydrazyl shown in eq 1 upon excitation $\left(\lambda_{\mathrm{exc}}=1064 \mathrm{~nm}\right)$ of the IVCT transition. Subsequent analysis of the scattering intensities, based on time-dependent scattering methods popularized by Heller ${ }^{12,13}$ and used by many groups, ${ }^{14-21}$ further yields a reorganization energy for each resonantly enhanced mode. These mode-specific reorganization energies are then used in a quantitative evaluation of Franck-Condon effects on the rate of thermally activated intramolecular electron transfer under idealized nonadiabatic conditions.

\section{Experimental Section}

The bis-hydrazyl dication, $\mathbf{2}^{2+}$, was synthesized and characterized by literature techniques as a tetrafluoroborate salt. ${ }^{22}$ Solutions for scattering experiments were made by dissolving the compound to millimolar levels in argon-saturated $\mathrm{CH}_{3} \mathrm{CN}$ (distilled over $\mathrm{CaH}_{2}$ ) containing $0.1 \mathrm{M}$ tetrabutylammonium hexafluorophosphate as a supporting electrolyte. Bulk electrochemical reduction was then carried out in an argon atmosphere at a potential of $-0.95 \mathrm{~V}$ (vs SSCE) in a three-chambered cell (glass frits separating the chambers) with a Pt mesh working electrode and a Pt wire counter electrode. As the radical cation was reductively formed, the solution changed from colorless to blue-green. It was then cannulaed into a standard NMR tube sealed with a rubber septum, where it was found to be stable for up to several hours. Decomposition was marked by a change of color from blue-green to light-yellow and by a lack of detectable scattering.

Solutions were excited either preresonantly by using a combination of mechanically chopped 1320 and $1337 \mathrm{~nm}$ excitation from a Nd:YAG laser or resonantly with $1064 \mathrm{~nm}$ radiation from the same laser. Laser powers were approximately $2 \mathrm{~W}$ at the shorter wavelength and $1.5 \mathrm{~W}$ at the (combined) longer wavelengths. The scattered light was then focused into a Spex 1403 double monochromator containing a pair of gratings blazed at $1.2 \mu \mathrm{m}$ (600 grooves $/ \mathrm{mm})$ and detected with a liquidnitrogen-cooled Ge photodiode (North Coast model EO817L)

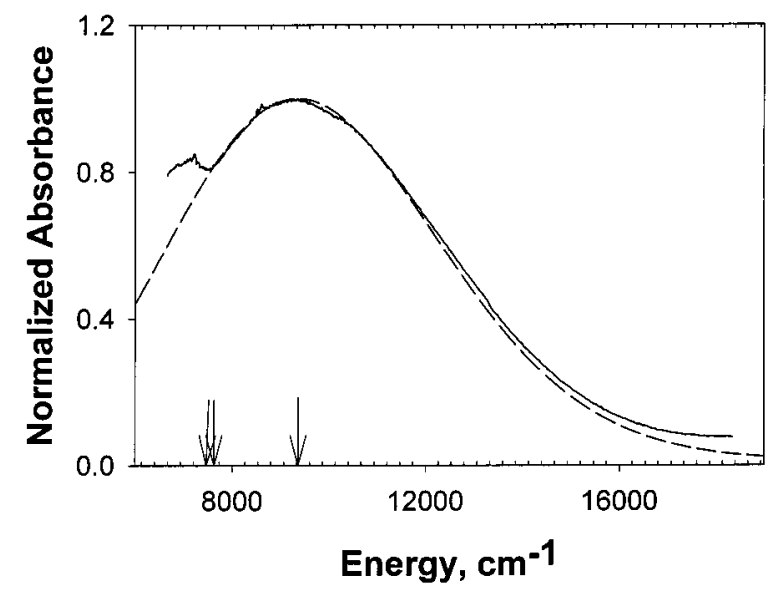

Figure 1. Near-infrared portion of the reduced bis-hydrazyl showing the intervalence absorption line shape (solid line) and the calculated fit (dashed line). Arrows mark the excitation energies used in the Raman scattering studies.

coupled to a lock-in amplifier. To prevent thermal decomposition during irradiation, the samples were cooled with a cold stream of nitrogen. Deleterious absorption of Raman scattered nearinfrared light by water vapor was avoided by purging the monochromator with dry nitrogen. ${ }^{11 \mathrm{~b}}$

\section{Results}

Spectra. The portion of the electronic absorbance spectrum associated with the IVCT for the syn isomer is shown in Figure 1 (absorption and scattering data for the anti isomer (which behaved similarly) are included as Supporting Information). Both the width and energy maximum in Figure 1 agree with previous reports. ${ }^{5}$ Interestingly, a prominent shoulder is found on the low-energy side of the main IVCT. This feature has also been noted previously ${ }^{5}$ and has been found to vary energetically in the same fashion as the main band when the solvent is varied. The secondary band is not an obvious consequence of vibrational substructure. Although the original report provided no explanation for the anamolous band, recent calculations ${ }^{9}$ have suggested that the pyramidality of the nitrogens may give rise to invertomers, with the low energy band arising from the less stable of the two forms. Whatever its origin, only the main IVCT band has been treated in this work.

Raman spectra were obtained in the $200-3500 \mathrm{~cm}^{-1}$ Raman shift region, although vibrational modes were found only in the 400-1600 $\mathrm{cm}^{-1}$ shift region. The Raman spectrum obtained with $1064 \mathrm{~nm}$ excitation (Figure 2) shows that six vibrations are enhanced by the intervalence transition. Although lack of isotopically substituted analogues precludes definitive assignments, determination of both the aza stretch $(-\mathrm{N}=\mathrm{N}-)^{+}$and its reduced counterpart $(-\mathrm{N}-\mathrm{N}-)^{\bullet}$ is of particular interest. The vibrations at 1220 and $1146 \mathrm{~cm}^{-1}$, respectively, are attractive candidates for several reasons. First, as would be expected for modes activated by moving an antibonding electron, they are the most intense in the spectrum. Second, their intensities are nearly equal. It should be noted, however, that $-\mathrm{N}=\mathrm{N}-$ vibrations in neutral molecules ${ }^{23}$ generally lie between 1400 and $1600 \mathrm{~cm}^{-1}$. The extra charge and reduced bond order in this system, however, might well reduce the frequencies of these modes by the necessary several hundred wavenumbers. One intriguing implication, if these assignments are correct, is that the excited-state frequencies for these modes are known; in the two-state diabatic limit, the $1146 \mathrm{~cm}^{-1}$ mode must have a frequency of $1220 \mathrm{~cm}^{-1}$ in the excited state and vice versa. 


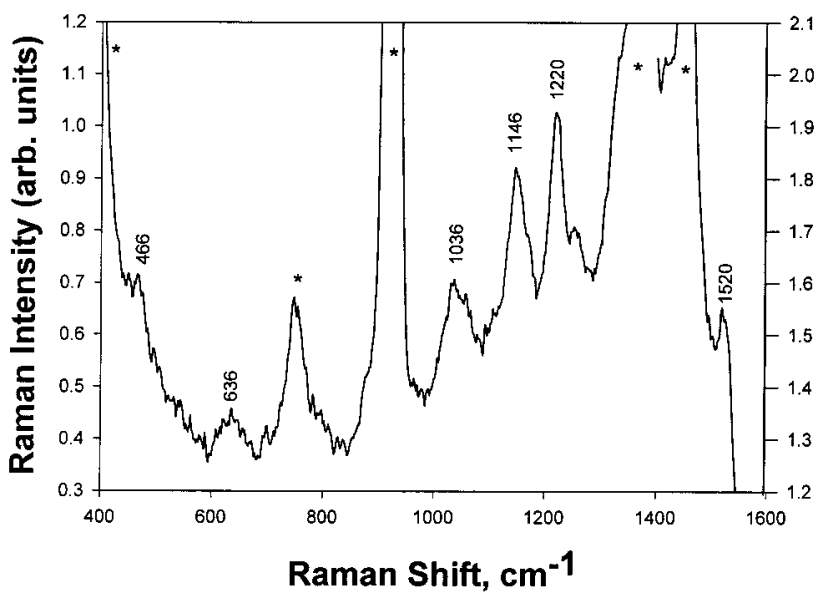

Figure 2. Resonance-enhanced Raman spectrum of the radical cation obtained with $1064 \mathrm{~nm}$ excitation. Starred peaks denote modes arising from the solvent. Note that the $1520 \mathrm{~cm}^{-1}$ mode is scaled to the righthand intensity axis.

TABLE 1: Frequencies, Corrected Intensities, and Franck-Condon Parameters for the Reaction Depicted in Eq 3

\begin{tabular}{cccc}
\hline $\begin{array}{c}\text { frequency } \\
\left(\mathrm{cm}^{-1}\right)\end{array}$ & $\begin{array}{c}\text { relative } \\
\text { scattering } \\
\text { intensity }\end{array}$ & $\Delta_{k}$ & $\chi_{k}{ }^{\prime}\left(\mathrm{cm}^{-1}\right)$ \\
\hline 466 & 0.18 & $1.64 \pm 0.09$ & $630 \pm 70$ \\
636 & 0.43 & $1.85 \pm 0.10$ & $1100 \pm 100$ \\
1036 & 0.47 & $1.18 \pm 0.06$ & $720 \pm 80$ \\
1146 & 0.81 & $1.40 \pm 0.07$ & $1130 \pm 120$ \\
1220 & 1.00 & $1.46 \pm 0.08$ & $1300 \pm 150$ \\
1520 & 0.36 & $0.70 \pm 0.04$ & $370 \pm 50$
\end{tabular}

Use of this information in the intensity analysis should, and does, result in convergence of the reorganization energies for these two modes, as would be required by the symmetry of the electronic transition.

In an effort to corroborate these assignments, we also performed nonresonant (1064 $\mathrm{nm}$ excitation) experiments on $\sim 1 \mathrm{M}$ acetonitrile solutions of the "monomer" form $\left(3^{+}\right)$of the mixed-valent molecule. The cationic species was found to have

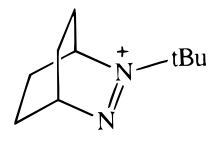

3

modes at 1183,1263 , and $1570 \mathrm{~cm}^{-1}$ (in addition to a number of lower frequency modes) that could correspond to the 1146 , 1220 , and $1520 \mathrm{~cm}^{-1}$ modes in the mixed-valent molecule. ${ }^{24}$ Unfortunately, we have been unable to prepare successfully by electrochemical techniques the corresponding neutral radical species in sufficient concentration for Raman characterization.

Enhanced spectra of the mixed-valent compound were also obtained on the basis of excitation with the $1320 / 1337 \mathrm{~nm}$ pair. In contrast, excitation with $514 \mathrm{~nm}$ light resulted in no detectable scattering, ${ }^{25}$ indicating that the near-infrared excited spectra are indeed resonantly enhanced. Another control experiment, implemented on a solution containing only the supporting electrolyte, indicated that scattering from $\mathrm{TBAPF}_{6}$ is undetectable at the concentrations used.

Relative Raman intensities (Table 1) were determined by correcting for instrument throughput and detector response (as outlined previously ${ }^{11 \mathrm{~b}}$ ) and then integrating the intensities and ratioing them to the most intense vibration (i.e., $1220 \mathrm{~cm}^{-1}$ ).
Self-absorption effects were taken into account by using the solvent-based $\mathrm{C}-\mathrm{C}$ stretch at $918 \mathrm{~cm}^{-1}$ as an internal intensity standard (see below). Admittedly this approach neglects any Raman shift dependence of the self-absorption correction. But given the extraordinary breadth of the absorption band and the proximity of the most important peak for $2^{2+}$ to the $918 \mathrm{~cm}^{-1}$ standard, neglect of the energy dependence of the correction is unlikely to be of substantial consequence.

Scattering Cross Sections. An absolute Raman scattering cross section for the $918 \mathrm{~cm}^{-1} \mathrm{C}-\mathrm{C}$ stretch in the $\mathrm{CH}_{3} \mathrm{CN}$ solvent was determined by extrapolation from a previous measurement obtained using ultraviolet excitation. ${ }^{26,27}$ With $1064 \mathrm{~nm}$ excitation, the cross section was estimated to be 4.7 $\times 10^{-15} \AA^{2}$. Cross sections for the resonance-enhanced solute modes were determined by comparing integrated intensities of each of the six modes to the solvent mode using known prescriptions. ${ }^{13 \mathrm{~b}}$ A depolarization ratio of 0.33 for the solute modes was assumed. The $1220 \mathrm{~cm}^{-1}$ mode was determined to have an absolute scattering cross section of $3.6 \times 10^{-12} \AA^{2}$. The molar extinction coefficient for the mixed-valence molecule of $1450 \mathrm{M}^{-1} \mathrm{~cm}^{-1}$ was taken from previous reports ${ }^{5}$ and converted to an absorption cross section of $0.055 \AA^{2}$ molecule ${ }^{-1}$ by using published procedures. ${ }^{13 \mathrm{~b}}$

Spectral Modeling. The measured Raman intensities are known to be functions of both (1) the frequencies and unitless displacements of each of the modes and (2) the various dephasing processes that occur in the excited state. ${ }^{15 a}$ If the potential energy surfaces for the ground and excited states are assumed to be harmonic with no Duschinsky rotation or coordinate dependence of the transition moment (Condon approximation), analytic expressions (corresponding to motion of Gaussian wave packets on the excited-state surface) may be employed to extract the unitless displacements. We note at the outset that an additional postulation (implicit in the assumption of a depolarization ratio of 0.33 for all of the modes) is that scattering arises from coupling of the ground state to a single excited state. Given the prominent shoulder found in the absorbance spectrum near the excitation wavelength (which might arise from an additional electronic transition), this postulation may be in error.

Since the absorption spectrum and Raman spectrum depend on the same potential energy surfaces, both are calculated. The absorption spectrum is calculated first in the time domain:

$$
\begin{aligned}
& A(t)=\exp \left(\sum _ { k } \left[-\frac{1}{2} \Delta_{k}{ }^{2}(1-\right.\right.\left.\left.\exp \left(-\mathrm{i} \omega_{k} \mathrm{t}\right)\right)\right]- \\
&\left.\mathrm{i}\left(E_{00}+\chi_{\mathrm{s}}\right) t-\left(\Gamma^{2}+\chi_{\mathrm{s}} k T\right) t^{2}\right)
\end{aligned}
$$

The conventional, frequency-domain absorption spectrum is obtained by a full Fourier transform of this expression as follows:

$$
A(\omega)=C \omega \int_{-\infty}^{\infty} A(t) \mathrm{e}^{\mathrm{i} \omega t} \mathrm{~d} t
$$

The shape and initial energy maximum of the absorbance spectrum arises (eq 4) from the summation over the $k$ Raman modes of frequency $\omega_{k}$ and unitless displacement $\Delta_{k}$. Terms involving the solvent reorganization $\left(\chi_{\mathrm{s}}\right)$ serve two purposes: (1) the imaginary term (i $\left.\chi_{\mathrm{s}} t\right)$ provides an energy shift that accounts for the Franck-Condon activity of the solvent and (2) the real term $\left(\chi_{\mathrm{s}} k T t^{2}\right.$, where $k T$ is taken to be $\left.200 \mathrm{~cm}^{-1}\right)$ introduces additional, solvent-induced broadening, which provides additional spectral diffuseness. ${ }^{28}$ The energy of the 
absorption maximum is increased by both $E_{00}$ (the energy gap between the vibrationally relaxed ground and excited states) and $\chi_{\mathrm{s}}$. The $\Gamma$ term accounts for additional spectral diffusion. In practice, this term often is employed as a fitting function that models neglected dephasing or spectral broadening phenomena (such as displacement of vibrational modes too weak to be observed directly by Raman scattering). In eq 5, $C$ is used as an arbitrary constant to normalize the absorbance spectrum.

Similar equations, previously published, ${ }^{11 \mathrm{a}, 12-21}$ are used to calculate the Raman excitation profile for each mode. In the interest of space, they are not repeated here.

Energy Parameters. As applied here, the spectral modeling ideally determines uniquely only the $\Delta_{k}$ 's and $\Gamma$, leaving both $\chi_{\mathrm{s}}$ and $E_{00}$ to be determined separately. Fortunately, the symmetry in this reaction nominally constrains $E_{00}$ to zero. ${ }^{29}$ There are several ways of estimating $\chi_{\mathrm{s}}$. According to Marcus, ${ }^{30}$ a linear correlation should exist between the optical intervalence energy maximum, $E_{\mathrm{op}}$, and a solvent dielectric factor related to $\chi_{\mathrm{s}}$. Unfortunately, since experimental measurements ${ }^{5}$ have not yielded such a correlation, a different approach was necessitated. If the effective charge-transfer distance is known, either a twosphere conductor model ${ }^{30}$ or an ellipsoidal cavity model ${ }^{31}$ can be employed to estimate $\chi_{\mathrm{s}}$ in a continuum-type solvent. The needed estimates for the charge-transfer distance were initially taken from AM1 calculated distances between the remote dinitrogen units and yielded, for a two-sphere model, a $\chi_{\mathrm{s}}$ of $3500 \mathrm{~cm}^{-1} .^{5}$ However, recent Stark spectroscopy studies of inorganic class II mixed-valence ions have shown that geometric arguments such as these may seriously overestimate the true charge-transfer distance. ${ }^{32}$ To gauge the magnitude of this problem here, we approximated the effective charge-transfer distance by determining Mulliken charge populations on each atom in the ground state using ZINDO. These Mulliken populations subsequently were used to determine an overall dipole moment for the ground state, ${ }^{33}$ a value that was doubled to give the ground-state/excited-state dipole moment difference (since, in the two-state limit, the excited state must have a dipole equal in magnitude but opposite in sign from the ground state). These calculations led to a dipole moment difference of $16 \mathrm{D}$, corresponding to transfer of a unit electronic charge through $3.3 \AA$ A. Using this value in an ellipsoidal cavity model (in which the major and minor axes for the ellipse ${ }^{34}$ were taken from the crystallography of the fully oxidized dication ${ }^{10}$ ) again yields a $\chi_{\mathrm{s}}$ of ca. $3500 \mathrm{~cm}^{-1}$, although the estimated uncertainty (based on how the ellipsoid parameters are chosen) is at least $500 \mathrm{~cm}^{-1}$.

Franck-Condon Parameters. Raman excitation profiles for each of the six modes were generated as described in the spectral modeling section using arbitrary values for the $\Delta_{k}$ 's. The $\Delta_{k}$ 's were subsequently adjusted iteratively until the calculated relative Raman intensities for each of the six modes matched the experimental intensities (at $1064 \mathrm{~nm}$ excitation) to within $0.5 \%$. These relative displacements were then used in eqs 3 and 4 to generate an absorbance spectrum. The calculated spectrum was then adjusted by both linearly scaling all of the $\Delta_{k}$ 's and adjusting $\Gamma$. The new values were then used to recalculate excitation profiles, and the entire process was iterated until a consistent fit to both types of spectra was achieved. Finally, the reorganization energy, $\chi_{k}{ }^{\prime}$, for each mode was calculated by using

$$
\chi_{k}^{\prime}=\frac{1}{2} \Delta_{k}^{2} \omega_{k}
$$

Figure 1 shows that a good fit to the intervalence absorbance was obtained, and Table 1 lists the associated parameters used.
Assuming that the assignments for the 1146 and $1220 \mathrm{~cm}^{-1}$ modes are valid, a dimensioned (units of $\AA$ ) displacement can also be estimated for these modes. Conversion of a unitless displacement to an absolute displacement entails knowledge of both the frequency and reduced mass of the oscillator in question. ${ }^{35}$ Taking a crude estimate of 7 for the reduced mass (correct for an isolated $\mathrm{N}=\mathrm{N}$ stretch) and a frequency and unitless displacement of $1220 \mathrm{~cm}^{-1}$ and 1.46 , respectively, yields a displacement of $0.09 \AA$. While this cannot be compared crystallographically to the radical mixed-valence species under study, the electron loss in this case is analogous to the second electron loss from tetraalkylhydrazines, since both involve conversion of a three-electron $\pi$ bond to a two-electron $\pi$ bond. $\mathrm{X}$-ray results for both electron losses are available only for a sesquibicyclo[2.2.2] octane system, ${ }^{36}$ for which the second electron loss leads to an $\mathrm{N}=\mathrm{N}$ bond shortening of $0.06 \AA$, in fair agreement with our $0.09 \AA$ value.

\section{Discussion}

Energy Parameters from Spectral Fits. Uniquely determining the $\Delta_{k}$ 's using eqs 3 and 4 is clearly dependent on having uniquely determined values for $E_{00}$ and $\chi_{\mathrm{s}}$. As stated above, $E_{00}$ has been constrained to zero and an independent measure of $\chi_{\mathrm{s}}$ has been used. Given the $\pm 500 \mathrm{~cm}^{-1}$ uncertainty in $\chi_{\mathrm{s}},{ }^{37}$ we executed several independent fits using the full range of its possible values. The reported lower limit (Table 1) for the $\Delta_{k}$ 's arises from fitting with the maximum value for $\chi_{\mathrm{s}}$ of $4000 \mathrm{~cm}^{-1}$, while the reported upper limit for the $\Delta_{k}$ 's arises from fitting with the minimum value for $\chi_{\mathrm{s}}$ of $3000 \mathrm{~cm}^{-1}$.

In the fitting, $\Gamma$ was initially set to $10 \mathrm{~cm}^{-1}$, reflecting its presumed origin in homogeneous "dephasing". We found, however, that for a $\chi_{\mathrm{s}}$ value of $3500 \mathrm{~cm}^{-1}$ a much larger value $\left(800 \mathrm{~cm}^{-1}\right)$ was needed to reproduce the absorption line shape adequately. With a $\chi_{\mathrm{s}}$ value of $3000 \mathrm{~cm}^{-1}$ a $\Gamma$ value of 700 $\mathrm{cm}^{-1}$ was required. Displaced vibrations at relatively high frequency not observed in the Raman spectrum could conceivably account for these findings. We find this unlikely, however, since any such vibration should be relatively intense. ${ }^{38,39}$ Another possibility is that the estimates for $\chi_{\mathrm{s}}$ are unreasonably high. Indeed, a good fit can be obtained by decreasing $\chi_{\mathrm{s}}$ to 2000 $\mathrm{cm}^{-1}$ and using a $\Gamma$ value of only $100 \mathrm{~cm}^{-1}$. A $\chi_{\mathrm{s}}$ value that small, however, would seem to require either a gross error in the geometric parameters used in the ellipsoidal cavity model or an even smaller charge-transfer distance than we have used. An additional explanation involving inhomogeneous broadening via incomplete ion pairing ${ }^{40}$ has (for acetonitrile, at least) been ruled out by earlier experiments. ${ }^{5}$

Absolute Raman Scattering Cross-Sections. As stated before, the energy needed to place the absorbance maximum correctly may come from either $\chi_{\mathrm{s}}$ or the $\Delta_{k}$ 's. In addition, since each of these terms $\left(\chi_{\mathrm{s}}\right.$ and $\left.\Delta_{k}\right)$ introduces spectral broadening in a subtly different way, redistributing the total energy between the two allows the absorbance line shape to be reproduced. For this particular system, when the $\Delta_{k}$ 's are increased (and $\chi_{\mathrm{s}}$ is decreased to offset the increased energy) the breadth of the absorbance spectrum is increased and a smaller value of $\Gamma$ may be used for the fitting. Conversely, increasing $\chi_{\mathrm{s}}$ and decreasing the $\Delta_{k}$ 's necessitate the use of larger $\Gamma$ values to achieve reasonable fits. To this point our supposition has been that fits requiring smaller $\Gamma$ values are physically more reasonable, since $\Gamma$ serves primarily as a fitting function.

In principle, another way of assessing how the total energy and spectral broadening are partitioned between the various parameters is to measure the absolute Raman scattering cross 
sections. The cross sections are sensitive to the partitioning between "homogeneous" broadening terms that interfere at the Raman amplitude level and "inhomogeneous" broadening terms that interfere at the Raman probability level. We could in principle use the Raman scattering equations, which are analogous to eqs 4 and 5, to calculate scattering cross sections (by using the appropriate physical constants in place of the normalization factor in eq 5). However, since all the terms in eq 4 , particularly $\chi_{\mathrm{s}}$, are treated as "homogeneous" broadening terms, repartitioning the total energy between these terms may not lead to large differences in calculated cross sections. A potentially more fruitful approach would involve adding several additional terms to the scattering and absorbance equations. First, adding an "inhomogeneous" spectral broadening term would provide a means to increase spectral diffusion without diminishing the scattering cross sections. Second, $\chi_{\mathrm{s}}$ could be incorporated in a way that recognizes that solvent spectral broadening may occur on different time scales, interpolating between making "homogeneous" and "inhomogeneous" contributions to the spectral line shape.

Using existing prescriptions ${ }^{13 b}$ and code,${ }^{41}$ we extended eqs 4 and 5 to include several other terms:

$$
\begin{aligned}
& \sigma_{A}(\omega)= \\
& \frac{5.75 \times 10^{-3} M^{2} \omega}{n} \int_{-\infty}^{\infty} \mathrm{d} \delta G(\delta) \sum_{i} B_{\mathrm{i}} \operatorname{Re} \int_{0}^{\infty} \mathrm{d} t\left\langle x_{i} \mid x_{i}(t)\right\rangle \times \\
& \exp \left[\mathrm{i}\left(\omega-E_{00}-\delta+\omega_{i}\right) t-g(t)\right]
\end{aligned}
$$

Here, $\sigma_{A}(\omega)$ is the absorption cross section in units of $\AA^{2 /}$ molecule, $M$ is the electronic transition length in $\AA$, and $G(\delta)$ is a normalized Gaussian distribution of frequency shifts centered at $\delta=0$. The overlap term in brackets takes account of mode frequencies and displacements in the same way as terms in eq 4 , while $B_{i}$ represents Boltzmann terms and Re designates the real part of the integral. The solvent reorganization energy is here accounted for using $g(t)$, a function that models the solvent as an overdamped Brownian oscillator. ${ }^{42}$

Whereas eqs 4 and 5 only took into account the "homogeneous" broadening terms found within the inner integral, the second integral in eq 7 allowed spectral broadening to be introduced through an "inhomogeneous" mechanism. We hoped that this additional fitting function, in combination with the more sophisticated Brownian oscillator model for the solvent, would allow us to simulate the Raman scattering cross sections successfully.

Reassuringly, we found that eq 7 (and its analogue for scattering cross sections) yielded good fits to both the experimental absorbance spectrum and to the relative Raman intensities when using the frequencies, displacements, $E_{00}$ value, and $\chi_{\mathrm{s}}$ value reported in Table 1 , with the Brownian oscillator in the Gaussian limit. ${ }^{43} \mathrm{We}$ also found, however, that the scattering cross sections calculated with these parameters were much higher (roughly an order of magnitude) than those measured experimentally. Clearly, the solution to this problem is not to partition some of the solvent energy into the inhomogeneous Gaussian distribution, $G(\delta)$, since this will increase-rather than decrease-all the calculated cross sections. ${ }^{44}$

Cross sections may be diminished by decreasing the mode displacements, increasing the homogeneous damping factor, or increasing the amount and/or type of solvent broadening. Increasing the displacements necessitated decreasing the solvent reorganization energy and the damping factor and in this case resulted in scattering cross sections that were close to those

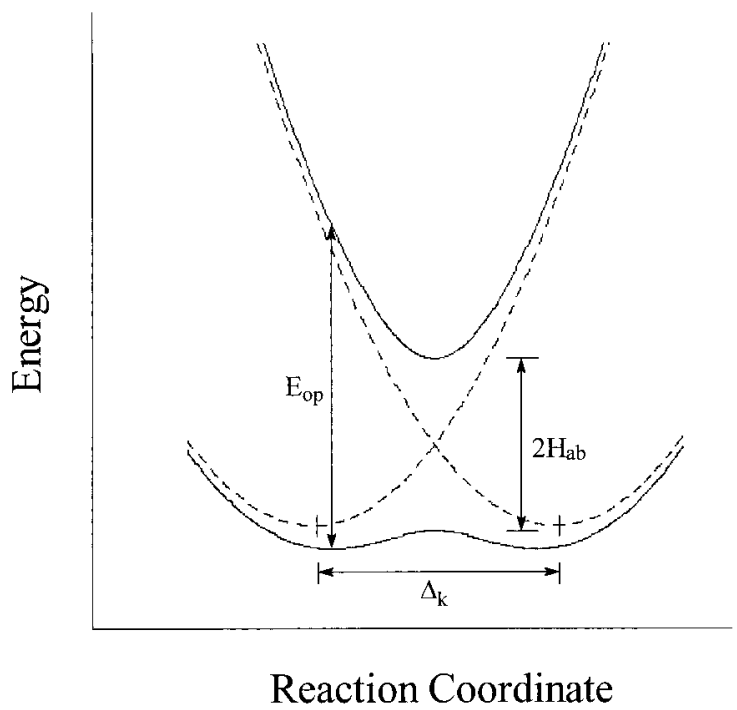

Figure 3. Adiabatic (solid line) and diabatic (dashed lines) potential energy surfaces for the mixed-valence cation, calculated assuming $H_{\mathrm{ab}}$ of $2500 \mathrm{~cm}^{-1}$ and $E_{\mathrm{op}}$ of $9400 \mathrm{~cm}^{-1}$.

calculated with the Table 1 values. Next, we attempted to use extremely large values for the homogeneous damping factor. These values, however, resulted in an extremely broad absorbance spectrum extending well into the visible region. The damping effect of $\chi_{\mathrm{s}}$ was then increased by using the Brownian oscillator in the Lorentzian limit. ${ }^{45}$ Unfortunately, this also resulted in an unphysically broad absorbance spectrum. In short, we found no consistent set of parameters that allowed us to reproduce both the absorbance spectrum and the scattering cross sections. We note finally, however, that coupling of the ground state to multiple excited states (recall the unidentified low-energy shoulder in the absorption spectrum) would also result in depressed cross sections. We have not investigated this possibility further.

Approximate Adiabatic Surface Propagation. One possible explanation for our inability to simulate successfully the absolute Raman cross sections stems from the unique nature of the potential surfaces for electronically symmetric systems. The scattering calculations are done on the diabatic surfaces shown as dashed lines in Figure 3, whereas the system actually evolves primarily on the upper (solid line) adiabatic surface. ${ }^{46}$ Conceptually, at least, the consequences of adiabatic wave packet propagation are profound. First, the upper and lower surfaces (adiabatic surfaces), in contrast to the right and left surfaces (diabatic surfaces) are not related simply as displaced, but otherwise equivalent, reaction surfaces. Second, the upper and lower surfaces, in contrast to the right and left surfaces, are inherently anharmonic. A rigorously correct treatment of the propagation problem, therefore, would require a relaxation of the frozen Gaussian wave packet assumption (since wave packet spreading will occur if propagation takes place anharmonically). ${ }^{47}$

We lack the machinery at present to implement a multidimensional, damped anharmonic wave packet propagation and overlap study. ${ }^{48}$ We reasoned, however, that some insight into the underlying Raman cross section problem might be gained by approximating the relevant upper and lower adiabatic surfaces with inequivalent, vertically displaced parabolic surfaces, as shown in Figure 4. If we assume that the wave packet is able to sample most or all of the region between the Franck-Condon region and the surface minimum, before suffering significant damping or dephasing, then a reasonable parabolic approximation might be one that makes the harmonic surface simulta- 


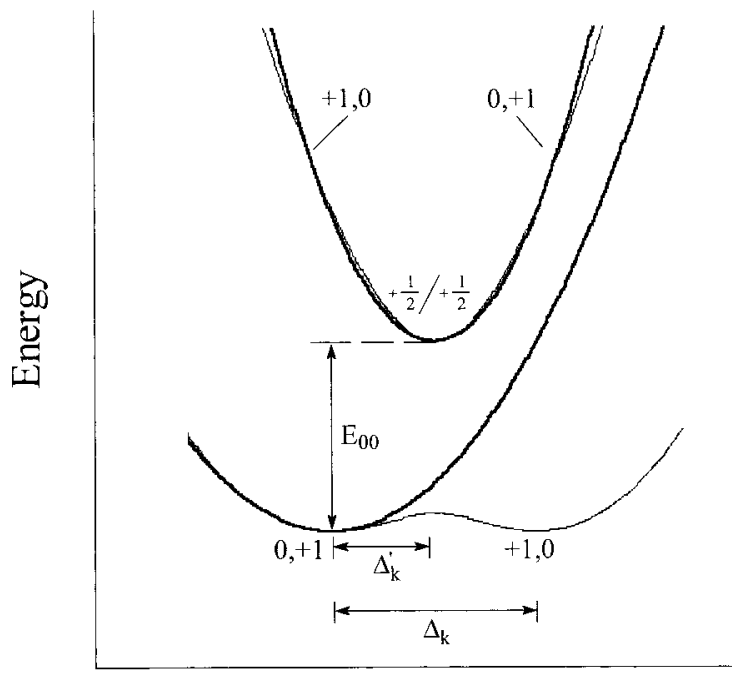

\section{Reaction Coordinate}

Figure 4. Adiabatic (light shading) and model harmonic (dark shading) surfaces. $E_{00}$ was taken to be $4850 \mathrm{~cm}^{-1}$.

neously coincident with the anharmonic surface in the FranckCondon and surface minimum regions. Under these conditions, we find for the bis-hydrazyl monocation that much larger upperstate frequencies (roughly 3 times the lower-state frequencies) are required (again, see Figure 4). Independent of other considerations, this effect alone leads to faster wave packet motion, diminished time-dependent initial-state (upper surface)/ static final-state (lower surface) vibrational overlap, and smaller calculated Raman scattering cross sections.

In Figure 4, however, the alternative modeling approach clearly introduces other changes. Most notably, an effective energy gap is generated. For illustrative computational purposes, we estimated the gap (i.e., the energy difference between the upper and lower surface minima) as $\left(E_{\mathrm{op}} / 4\right)+H_{\mathrm{ab}}$, where $H_{\mathrm{ab}}$ is the estimated initial-state/final-state electronic coupling energy. ${ }^{49}$ The value obtained was $4050 \mathrm{~cm}^{-1}$.

The alternative modeling approach also suggests a smaller apparent solvent reorganization energy. The reasoning is the following. Intervalence excitation converts the $0 /+$ form of the bis-hyrazyl cation into the vibrationally excited $+/ 0$ form. Progress along the upper surface, however, yields at the surface minimum the delocalized form $\left(+1 / 2 /+^{1} / 2\right)$. (Curve crossing, on the other hand, would yield ultimately the vibrationally relaxed +/0 form (right-hand side of lower potential energy surface; see Figure 4).) Since the solvent reorganization energy is expected to scale as the square of the amount of charge transferred, relocation from either minimum on the lower potential energy surface to the single minimum on the upper potential energy surface should entail only about one-fourth as much solvent reorganization as needed in the full charge-transfer case. (Similarly, migration from the Franck-Condon region of the upper surface $(0 /+$ region) to the minimum energy region $(+1 / 2 /+1 / 2$ region) should require dispersion of only about onefourth of the previously estimated solvent reorganization energy.) For modeling purposes, we have used an alternative $\chi_{\mathrm{s}}$ value of $900 \mathrm{~cm}^{-1}$.

Finally, note from Figure 4 that the displacements obtained from the alternative modeling (labeled $\Delta_{k}{ }^{\prime}$ ) are initially expected to be only half as large as the $\Delta_{k}$ values needed for full intramolecular electron transfer. In addition, it should be noted that the $\Delta_{k}$ values are only dimensionless when the groundand excited-state frequencies are degenerate; otherwise, the displacements used in the calculation $\left(\Delta_{k}{ }^{\prime \prime}\right)$ differ from $\Delta_{k}{ }^{\prime}$ by the factor $\left(\omega_{\text {excited }} / \omega_{\text {ground }}\right)^{-1 / 2} .50$

Our initial simulations of the adiabatic surfaces, then, used an $E_{00}$ value of $4050 \mathrm{~cm}^{-1}$, a $\chi_{\mathrm{s}}$ value of $883 \mathrm{~cm}^{-1}$ (input using the Brownian oscillator method ${ }^{13 b}$ outlined earlier with a $\kappa$ of 0.1 to provide Gaussian broadening), upper state frequencies 3 times larger than the ground-state (Raman determined) frequencies, and $\Delta_{k}^{\prime \prime}$ values obtained from halving the $\Delta_{k}$ values reported in Table 1 and then applying the $3^{-1 / 2}$ correction value (e.g., $\Delta^{\prime \prime}$ for the $466 \mathrm{~cm}^{-1}$ mode was 0.55$) .51,52$ The results of this simulation are worthy of comment. First, the scattering cross sections indeed do decrease by about an order of magnitude. Second, the absorbance spectrum now possesses considerable vibrational structure, especially at lower energy, presumably arising from the difficulty in damping wave packet motion on the high-frequency upper surface. Third, the calculated spectrum still extends unrealistically into the visible region of the spectrum and does not display the correct absorbance maximum. The absorbance could be shifted to lower energy by lowering further the displacements, although this does not change appreciably the spectral line shape.

Although this approach to modeling adiabatic surfaces clearly is not robust enough to provide justifiable, quantitative estimates of all of the relevant parameters, we believe it demonstrates rather convincingly that the low scattering cross sections may arise from a combination of effects whose origin lies in the unique shapes of the surfaces. Regardless of the explanation for this system, it is possible that the wave packet methodology we used initially may not be sufficient to calculate accurately cross sections for electronically symmetrical systems. We are currently investigating an alternative approach that we believe will eventually allow incorporation of the relevant effects to permit further examination of these systems.

Electron-Transfer Kinetics: Idealized Nonadiabatic Rate Calculations. The available mode-specific reorganizational information lends itself well to the calculation of ET reaction barriers in a fully quantum mechanical sense. Indeed, standard nonadiabatic, Golden Rule type approaches to electron transfer rate calculations require a summation over all vibrational modes that couple the initial and final electronic states. One interesting outcome of the resonance Raman study is that all of the vibrations coupled to the electron transfer are found at frequencies greater than $k T$, and the most important (in a reorganization energy sense) lies above $1000 \mathrm{~cm}^{-1}$. In the case of limited displacements, high-frequency vibrational modes generally lead to more efficient coupling (i.e., greater overall Franck-Condon overlap) and faster electron-transfer rates than do otherwise equivalent low-frequency (classical) modes. We have also undertaken an analysis of the anticipated influence of each particular mode on the overall rate of the thermal electrontransfer reaction (eq 3). ${ }^{11 a, 53}$

For simplicity, we have evaluated the rate effects under hypothetical nonadiabatic conditions; i.e., we have neglected the likely substantial effects of barrier "round off" and surface anharmonicity on the kinetics and dynamics of the reaction depicted in eq 3. To implement this, we first calculated a rate based on the "classical" (activated barrier crossing) equation"

$$
k=\frac{H_{\mathrm{ab}}^{2}}{\hbar}\left(\frac{\pi}{\chi_{\mathrm{tot}} R T}\right)^{1 / 2} \exp \left(\frac{-\chi_{\mathrm{tot}}}{4 \mathrm{RT}}\right)
$$

in which $\chi_{\text {tot }}$ is the total reorganization energy (the sum of $\chi_{\mathrm{s}}$ and all of the $\chi_{k}$ 's). We then calculated a rate based on an 
TABLE 2: Rate Parameters Determined Using Eqs 8 and 9 for the Reaction Depicted in Eq 3

\begin{tabular}{cccc}
\hline $\begin{array}{c}\text { frequency } \\
\left(\mathrm{cm}^{-1}\right)\end{array}$ & $\chi_{k}{ }^{\prime}\left(\mathrm{cm}^{-1}\right)$ & $\begin{array}{c}\text { rate } \\
\text { attenuation } \\
\text { factor }\end{array}$ & $\begin{array}{c}\text { nuclear } \\
\text { tunneling } \\
\text { factor }\end{array}$ \\
\hline 466 & 630 & 2.1 & 1.1 \\
636 & 1100 & 3.2 & 1.3 \\
1036 & 720 & 1.9 & 1.4 \\
1146 & 1130 & 2.4 & 1.8 \\
1220 & 1300 & 2.7 & 2.0 \\
1520 & 370 & 1.3 & 1.3 \\
total & $5250^{a}$ & $110^{b}$ & $9^{b}$ \\
${ }^{a}$ Total obtained as sum. ${ }^{b}$ Total obtained as product.
\end{tabular}

equation that accounts for mode-specific tunneling corrections to the rate:

$$
k=\frac{H_{\mathrm{ab}}^{2}}{\hbar}\left(\frac{\pi}{\chi_{\mathrm{s}} R T}\right)^{1 / 2} f(F C)
$$

In this equation, $f(F C)$ takes account ${ }^{54}$ of both the frequency and unitless displacement of each of the coupled modes. ${ }^{55}$ To avoid errors associated with $H_{\mathrm{ab}}$, we have used an arbitrary value for $H_{\mathrm{ab}}$ in each of the equations (both the classical and the vibrational mode specific) and have taken a ratio of the two rates. The calculated ratio proved to be comparatively large (ca. 10), indicating that the high-frequency vibrations collectively exert a considerable nonclassical or "nuclear tunneling" effect upon the rate.

Several additional calculations were performed to ascertain which of the vibrations provided the largest kinetic effects. To start, a new rate was calculated with a particular vibration omitted completely. A ratio of that rate to the rate calculated with all six vibrations included determines the kinetic importance of the omitted mode (rate attenuation factor in Table 2). Another rate was subsequently calculated with the reorganization energy (from eq 5) of the omitted mode added to $\chi_{\mathrm{s}}$ to keep the total reorganization energy constant. A ratio of that rate to the original rate quantifies a nuclear tunneling factor or nonclassical rate enhancement factor for that mode. These calculations, summarized in Table 2, indicate that the $1220 \mathrm{~cm}^{-1}$ mode (which has the greatest reorganization energy) has nearly the greatest rate attenuation factor and the greatest nuclear tunneling factor. The $466 \mathrm{~cm}^{-1}$ vibration (with the second smallest reorganization energy) has the smallest tunneling factor, while the $1520 \mathrm{~cm}^{-1}$ mode, which is only very slightly displaced, provides the least rate attenuation. Finally, we find the product of the rate attenuations to be equal to the overall rate ratio that was calculated initially (as expected, if the analysis has been implemented correctly).

Additionally, activation parameters for both the quantum and the classical cases may be determined by calculating rates as a function of temperature. A plot of $\ln \left(k_{\mathrm{et}} T^{-2}\right)$ against $1 / T$ over a temperature range $240-300 \mathrm{~K}$ reveals a linear relation with a slope that corresponds to an Arrhenius type activation enthalpy $\left(\Delta H^{*}\right)$. An activation enthalpy of $26 \mathrm{~kJ} \mathrm{~mol}^{-1}$ was determined in this manner for rates that were calculated fully classically (eq 7), whereas an activation enthalpy of only $12 \mathrm{~kJ} \mathrm{~mol}^{-1}$ was found using rates that were calculated with the quantum mechanical rate values (eq 9). ${ }^{56}$ Combining $\Delta H^{*}$ with $k_{\text {et }}$ and the appropriate nonadiabatic prefactor (see eq 9) yields an apparent activation entropy for the quantum case that is $-29 \mathrm{~J}$ $\mathrm{K}^{-1} \mathrm{~mol}^{-1}$ (at $298 \mathrm{~K}$ ), whereas in the classical case (since redoxinduced changes in the vibrational frequencies are ignored) the activation entropy would be zero. We postulate that nonclassical

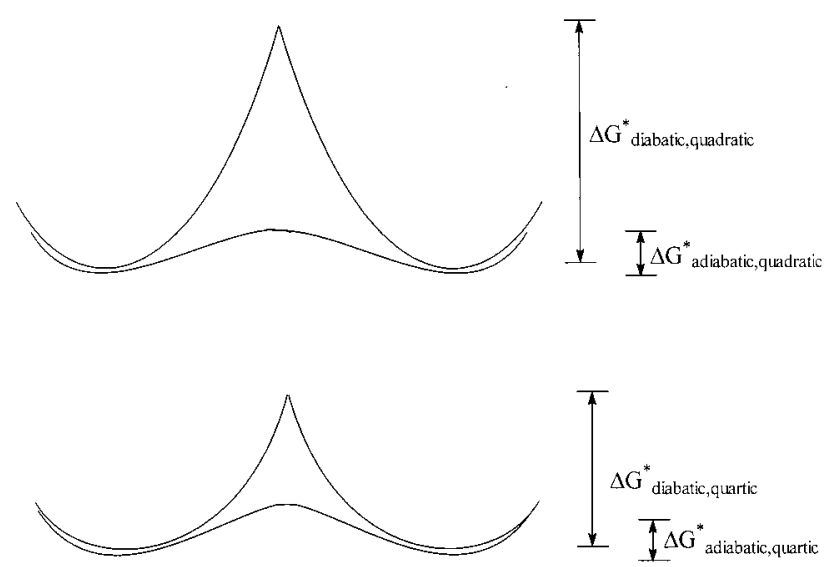

Figure 5. Adiabatic and diabatic representations of the conventional, quadratic surfaces are shown on top. Adiabatic and diabatic representations of the "quartic augmented" surfaces are down on bottom. $\Delta G^{*}$ for all the surfaces have been highlighted. These surfaces are illustrative only and have not been drawn analytically.

rate effects due to high-frequency modes might best be uncovered by examination of the temperature dependence of the rate.

Adiabatic Rate Calculations. Finally, one of us has reported recently on the use of "quartic augmented" surfaces for the calculation of rates of electron transfer using an adiabatic rate expression. ${ }^{8}$ Addition of a quartic term to the (usually) quadratic diabatic surfaces effectively lowers the barrier to thermal electron transfer $\left(\Delta G^{*}\right)$. This barrier diminution was found to be necessary ${ }^{8}$ in order to decrease the calculated rate of electron transfer to the rate that is experimentally measured for the $6 \sigma$ bonded hydrazine system under study. ${ }^{6,57}$ A similar issue may, in principle, be operative in this case. We hypothesized, however, that using the usual quadratic surfaces in concert with the ZINDO-derived $H_{\mathrm{ab}}$ value ${ }^{49}$ would yield a reasonable adiabatic rate. In fact, it should be similar to the rate obtained using quartic augmented surfaces along with the smaller $H_{\mathrm{ab}}$ value reported previously (see Figure 5). ${ }^{5}$ To test this notion, adiabatic rates were calculated by using the expression ${ }^{8}$

$$
k_{\mathrm{et}}=v_{v}\left(\frac{\chi_{v}}{\chi_{\text {total }}}\right)^{1 / 2} \exp \left(-\frac{\Delta G^{*}}{R T}\right)
$$

where $v_{v}$ is a weighted average vibrational frequency ${ }^{58}$ and $\chi_{v}$ is the total vibrational reorganization energy. Using a $\Delta G^{*}$ value of $1000 \mathrm{~cm}^{-1}$ (obtained ${ }^{59}$ by using an $H_{\mathrm{ab}}$ value of $1200 \mathrm{~cm}^{-1}$ with the quartic augmented surfaces) yields a rate of $1.8 \times 10^{11}$ $\mathrm{s}^{-1}$, while a $\Delta \mathrm{G}^{*}$ value of $960 \mathrm{~cm}^{-1}$ (obtained from the 1700 $\mathrm{cm}^{-1} H_{\mathrm{ab}}$ value together with initially quadratic surfaces) yields a rate of $2.2 \times 10^{11} \mathrm{~s}^{-1}$.

\section{Conclusions}

Resonance Raman scattering studies of an organic radical cation revealed six vibrations coupled to an intramolecular charge transfer transition between aza type components of the molecule. The two strongest vibrations have been tentatively assigned to vibrations of the dinitrogen units. Analysis of both the relative Raman intensities and the absorbance spectrum using time-dependent scattering theories indicated that all six modes make contributions to the vibrational reorganization energy, with the dinitrogen stretches being the most important. Dielectric continuum theories have been used in conjunction with crystallographic measurements and ZINDO calculations to estimate the reorganization energy contributed by the solvent. Spectral 
analyses based on relative Raman intensities indicated that unrealistically large homogeneous dephasing factors are needed in order to reproduce the experimental absorbance lineshape. A similar analysis based on absolute Raman scattering cross sections showed that the experimental Raman intensities are much smaller than predicted by the simplest forms of the analysis probably because of complications due to anharmonic (adiabatic) surface propagation. An examination of the nonclassical kinetic effects of the vibrational modes coupled to the electron transfer indicated that the modes collectively exert a considerable influence on the rate, while a mode-by-mode examination confirmed that both the frequency and the extent of displacement of a given mode are important factors in defining its effect upon the ET rate.

Acknowledgment. We thank Prof. Anne Myers for helpful discussions as well as access to a program for calculating absolute scattering cross sections and Prof. Michael Wasielewski for helpful discussions regarding vibrational assignments. We also acknowledge Fred Vance for help with the $\Delta \mu / \chi_{\mathrm{s}} /$ Mulliken population calculations. We additionally thank the U.S. Department of Energy, Office of Energy Research, Division of Chemical Sciences (Grant No. DE-FG02-87ER13808 for J.T.H.) and the NSF (Grant No. 9417946 for S.F.N.; graduate traineeship for R.D.W.) for support of this work.

Supporting Information Available: Resonance Raman and near-infrared intervalence absorbance spectra of the anti isomer and a table containing intervalence absorbance fitting parameters for the anti isomer. This material is available free of charge via the Internet at http://pubs.acs.org.

\section{References and Notes}

(1) Schroeder, A. H.; Mazur, S. J. Am. Chem. Soc. 1978, 100, 7339.

(2) Creutz, C. Prog. Inorg. Chem. 1983, 30, 1.

(3) (a) Hush, N. S. Coord. Chem. Rev. 1985, 64, 135. (b) Hush, N. S. Prog. Inorg. Chem. 1967, 8, 391.

(4) (a) Sedo, J.; Ruiz, D.; Vidal-Gancedo, J.; Rovira, C.; Bonvoisin, J.; Launay, J.-P.; Veciana, J. Synth. Met. 1997, 85, 1651. (b) Lahlil, K.; Moradpour, A.; Bowlas, C.; Menou, F.; Cassoux, A.; Bonvoisin, J.; Launay, J.-P.; Dive, G.; Dehareng, D. J. Am. Chem. Soc. 1995, 117, 9995. (c) Rak, S. F.; Miller, L. L. J. Am. Chem. Soc. 1992, 114, 1388. (d) Utampanya, S.; Rajca, A. J. Am. Chem. Soc. 1991, 113, 9242. (e) Mazur, S.; Sreekumar, C.; Schroeder, A. H. J. Am. Chem. Soc. 1976, 98, 6713. (f) Wudl, F.; Wobschall, D.; Hufnagel, E. J. J. Am. Chem. Soc. 1972, 94, 670.

(5) Nelsen, S. F.; Chang, H.; Wolff, J. J.; Adamus, J. J. Am. Chem. Soc. 1993, 115, 12276 .

(6) Nelsen, S. F.; Ramm, M. T.; Wolff, J. J.; Powell, D. R. J. Am. Chem. Soc. 1997, 119, 6863.

(7) Nelsen, S. F.; Ramm, M. T.; Wolff, J. J.; Powell, D. R. J. Org. Chem. 1996, 61, 4703. 846.

(8) Nelsen, S. F.; Ismagilov, R.; Trieber, D. A. Science 1997, 278,

(9) Nelsen, S. F. J. Am. Chem. Soc. 1996, 118, 2047.

(10) Nelsen, S. F.; Chang, H.; Wolff, J. J.; Powell, D. R. J. Org. Chem. 1994, 59, 6558.

(11) (a) Williams, R. D.; Petrov, V. I.; Lu, H. P.; Hupp, J. T. J. Phys. Chem. A 1997, 101, 8070. (b) Lu, H.; Petrov, V.; Hupp, J. T. Chem. Phys. Lett. 1995, 235, 521. (c) Petrov, V.; Hupp, J. T.; Mottley, C.; Mann, L. C. J. Am. Chem. Soc. 1994, 116, 2171. (d) Doorn, S. K.; Hupp, J. T. J. Am. Chem. Soc. 1989, 111, 1142. (e) Clark, R. J. H.; Dines, T. J. Angew. Chem., Int. Ed. Engl. 1986, 25, 131.

(12) (a) Heller, E. J.; Sundberg, R. L.; Tannor, D. J. Phys. Chem. 1982, 86, 1822. (b) Tannor, D. J.; Heller, E. J. J. Chem. Phys. 1982, 77, 202. (c) Heller, E. J. Acc. Chem. Res. 1981, 14, 368. (d) Lee, S.-Y.; Heller, E. J. J. Chem. Phys. 1979, 71, 4777.

(13) (a) Myers, A. B. Acc. Chem. Res. 1997, 30, 519. (b) Myers, A. B. in Laser Techniques in Chemistry; Myers, A. B., Rizzo, T. R., Eds.; Techniques of Chemistry 23; Wiley: New York, 1995; p 325. (c) Myers, A. B. Chem. Rev. 1996, 96, 911. (d) Myers, A. B. Chem. Phys. 1994, 180, 215. (e) Shin, K. S. K.; Zink, J. I. Adv. Photochem. 1991, 16, 119. (f) Myers, A. B.; Mathies, R. A. In Biological Applications of Raman Spectroscopy; Spiro, T. G., Ed.; Wiley: New York, 1987; Vol. 2, pp 1-58.
(14) (a) Phillips, D. L.; Gould, I. R.; Verhoeven, J. W.; TittelbachHelmrich, D.; Myers, A. B. Chem. Phys. Lett. 1996, 258, 87. (b) Kulinowski, K.; Gould, I. R.; Myers, A. B. J. Phys. Chem. 1995, 99, 9017. (c) Kulinowski, K.; Gould, I. R.; Ferris, N. S.; Myers, A. B. J. Phys. Chem. 1995, 99, 17715.

(15) (a) Cherepy, N. J.; Shreve, A. P.; Moore, L. J.; Boxer, S. G.; Mathies, R. A. J. Phys. Chem. B 1997, 101, 3250. (b) Shreve, A. P.; Mathies, R. A. J. Phys. Chem. 1995, 99, 7285. (c) Reid, P. J.; Shreve, A. P.; Mathies, R. A. J. Phys. Chem. 1993, 97, 12691. (d) Loppnow, G. R.; Mathies, R. A. Biophys. J. 1988, 54, 35. (e) Myers, A. B.; Harris, R. A.; Mathies, R. A. J. Chem. Phys. 1983, 79, 603

(16) (a) Wooton, J. L.; Zink, J. I. J. Am. Chem. Soc. 1997, 119, 1895. (b) Hanna, S.; Zink, J. I. Inorg. Chem. 1996, 35, 297. (c) Wooton, J. L.; Zink, J. I. J. Phys. Chem. 1995, 99, 7251. (d) Zink, J. I.; Reber, C. Coord. Chem. Rev. 1991, 111, 114. (e) Shin, K.-S. K.; Clark, R. J. H.; Zink, J. I. J. Am. Chem. Soc. 1990, 112, 3754.

(17) Britt, B. M.; McHale, J. L.; Friedrich, D. M. J. Phys. Chem. 1995 99, 6347.

(18) Davis, M. J.; Reber, C. Inorg. Chem. 1995, 34, 4585.

(19) (a) Loppnow, G. R.; Fraga, E. J. Am. Chem. Soc. 1997, 119, 896 (b) Fraga, E.; Webb, M. A.; Loppnow, G. R. J. Phys. Chem. 1996, 100, 3278.

(20) Esposito, A. P.; Foster, C. E.; Beckman, R. A.; Reid, P. J. J. Phys. Chem. A 1997, 101, 5309.

(21) Kwok, W. M.; Phillips, D. L.; Yeung, P. K.; Yan, V. W. J. Phys. Chem. A 1997, 101, 9286.

(22) Two different isomers were prepared and studied separately: the syn isomer shown in eq 1 and an anti isomer in which the second tertbutyl group is found on the nitrogen furthest from that on which the first tert-butyl is found. Qualitatively similar results were found for the anti but have not been analyzed in detail. The Raman spectrum and the intervalence absorbance line shape of the anti isomer, along with a spectral fit, are reported in the supplemental section of this work.

(23) (a) Lin-Vien, D.; Colthup, N. B.; Fateley, W. G.; Grasselli, J. G. Infrared and Raman Characteristic Frequencies of Organic Molecules; Academic Press: San Diego, CA, 1991. (b) Ackermann, M. N.; Kou, L.J.; Richter, J. M.; Willett, R. M. Inorg. Chem. 1977, 16, 1298. (c) Cohen, S. G.; Zand, R. J. Am. Chem. Soc. 1962, 84, 586.

(24) $\mathrm{Ab}$ initio calculations performed at the B3LYP/6-31G* level indicated that an IR-active " $\mathrm{N}=\mathrm{N}$ " stretch is expected at $1642 \mathrm{~cm}^{-1}$ in $\mathbf{3}^{+}$. This particularly high value provides contrary evidence for assigning the 1146 and $1220 \mathrm{~cm}^{-1}$ Raman modes in $\mathbf{2}^{+}$to the $\mathrm{N}=\mathrm{N}$ and $\mathrm{N}-\mathrm{N}$ stretches, respectively. Note, however, that the normal mode descriptions of the 1146 and $1220 \mathrm{~cm}^{-1}$ vibrations almost certainly are more complicated than assignment to a "pure" stretch would imply.

(25) Visible region experiments were implemented using an $\mathrm{Ar}^{+}$laser, Spex Triplemate monochromator, and CCD detection.

(26) Dudik, J. M.; Johnson, C. R.; Asher, S. A. J. Chem. Phys. 1985, 82,1732 .

(27) Several potential problems with this extrapolation (in which $A$-term fit parameters determined experimentally in ref 26 were used) should be noted. The first is that $1064 \mathrm{~nm}$ is located considerably further to the red than the $514 \mathrm{~nm}$ excitation point that was the furthest red point measured in ref 26. However, one would not expect a great difference in preresonance enhancement between 514 and $1064 \mathrm{~nm}$. A more serious error may potentially arise from measurements in ref 26 having been taken in aqueous acetonitrile rather than the pure liquid. Li and Myers (J. Phys. Chem. 1990, 94 , 4051) measured a cross section for the $2200 \mathrm{~cm}^{-1} \mathrm{CN}$ stretch (not measured in our experiments) in pure liquid that was $50 \%$ higher than the value obtained in aqueous solution. Similar problems may exist for the 918 $\mathrm{cm}^{-1} \mathrm{CC}$ stretch.

(28) As elucidated in ref $13 \mathrm{~d}$, the time-dependent $\chi_{\mathrm{s}}$ terms correspond to a high temperature, slow-modulation limit of stochastic theory and are equivalent in the frequency domain to convolution with a Gaussian line shape.

(29) As discussed in detail in ref $11 \mathrm{a}, E_{00}$ (as applied here) is strictly only zero in the diabatic limit. Given the uncertainty related to the magnitude of electronic coupling, we have left $E_{00}$ at zero for purposes of spectral simulations.

(30) Marcus, R. A. J. Chem. Phys. 1965, 43, 679.

(31) Brunschwig, B. S.; Ehrenson, S.; Sutin, N. J. Phys. Chem. 1986, 90, 3657.

(32) Unfortunately, we have been unable to apply Stark spectroscopy to these systems because of both their air sensitivity and the need for supporting electrolyte (which often precludes dielectrically stable glass formation). For reviews of applications of this technique to other class II ions, see the following. (a) Bublitz, G. U.; Boxer, S. G. Annu. Rev. Phys. Chem. 1997, 48, 213. (b) Vance, F. W.; Williams, R. D.; Hupp, J. T. Int. Rev. Phys. Chem. 1998, 17, 307-329.

(33) While there are potential difficulties in defining dipole moments for charged species, the difference in dipole moment (ground state vs excited state) is a well-defined quantity and, therefore, is invariant with changes in coordinate system or changes in counterion distribution. 
(34) A long axis of $14 \AA$, a short axis of $7 \AA$, and a charge-transfer distance of $3.3 \AA$ were used in an ellipsoidal cavity to generate a $\chi_{\mathrm{s}}$ of $3100 \mathrm{~cm}^{-1}$.

(35) Conversion was made by using the following equation (where $\delta$ is the dimensioned displacement and $\Delta$ is dimensionless) found in ref $13 \mathrm{e}$ :

$$
\delta=\sqrt{\frac{6.023 \times 10^{23}}{m} \frac{\hbar}{2 \pi c \omega}} \times 10^{8} \times \Delta
$$

(36) Nelsen, S. F.; Frigo, T. B.; Kim, Y.; Thompson-Colón, J. A. J. Am. Chem. Soc. 1986, 108, 7926.

(37) The most accessible continuum models for $\chi_{\mathrm{s}}$ are those that assume either spherical (e.g., $\mathrm{Fe}\left(\mathrm{H}_{2} \mathrm{O}\right)_{6}{ }^{2+}$ ) or elliptical (e.g., the Creutz-Taube ion) shapes. Since the species under investigation here possesses neither, it is not surprising that estimates based on parametrizing an ellipse or sphere to contain the molecule yield considerable uncertainties in $\chi_{\mathrm{s}}$.

(38) To a first approximation, the resonance Raman intensity scales as $\left(\omega_{k} \Delta_{k}\right)^{2}$ so that large intensities are expected for large Raman shifts (although strictly only for frequencies less than $\Gamma$ ).

(39) Low-frequency vibrations are (probably) missed quite frequently in frequency-domain Raman experiments because of their low intensity and their coincidence with Rayleigh scattering. However, the reorganization energies for these vibrations tend to be hidden in $\chi_{\mathrm{s}}$ and are not high enough in frequency to mimic the large $\Gamma$ values that we have obtained.

(40) This possibility has been excluded, since the absorbance line shape showed no dependence on counterion identity or concentration. ${ }^{5}$ For a discussion of the effects of partial and complete ion pairing upon intervalence absorption line shapes and energies, see the following. Blackbourn, R. L.; Hupp, J. T. J. Phys. Chem. 1990, 94, 1788.

(41) FORTRAN code for calculating absolute cross sections was obtained courtesy of Prof. Anne Myers at the University of Rochester.

(42) The equation is:

$$
g(t)=\left(\frac{D}{\Lambda}\right)^{2}\left(\Lambda t-1+\mathrm{e}^{-\Delta t}\right)+\mathrm{i}\left(\frac{D^{2}}{2 k T \Lambda}\right)\left(1-\mathrm{e}^{-\Gamma t}\right)+\frac{t}{\tau}
$$

where $T$ is the temperature and $D$ and $\Lambda$ are used to describe the characteristic magnitude and frequency of the solvent perturbation, respectively. In this equation, the real part contributes spectral broadening while the imaginary part contributes the Franck-Condon shift.

(43) In addition to the Table 1 values for the frequencies and displacements, a transition length of $0.84 \AA$ was used with a lifetime (analogous to $\Gamma$ in eq 4) broadening value of $700 \mathrm{~cm}^{-1}$. Parameters for the Brownian oscillator included a $\kappa$ (line shape parameter) of 0.01 (Gaussian limit) and a $\lambda$ of 2600 , resulting in a $\chi_{\mathrm{s}}$ of $2949 \mathrm{~cm}^{-1}$. These parameters yielded a cross section for the $1220 \mathrm{~cm}^{-1}$ mode of $2.7 \times 10^{-11} \AA^{2} /$ molecule.

(44) We did perform a calculation in which $\chi_{\mathrm{s}}$ was partitioned into the inhomogeneous term. In addition to Table 1 values for the frequencies and displacements, an $E_{00}$ of $2700 \mathrm{~cm}^{-1}$ (to model the Franck-Condon shift that the solvent would contribute), an inhomogeneous broadening term of $2700 \mathrm{~cm}^{-1}$ (standard deviation), a homogeneous (lifetime) broadening of $700 \mathrm{~cm}^{-1}$, and a transition length of $0.95 \AA$ were used. There was no $\chi_{\mathrm{s}}$ term in this calculation. These parameters resulted in an absorbance spectrum that was somewhat broader than the experimental spectrum but with cross sections $\left(4.4 \times 10^{-11} \AA^{2} /\right.$ molecule for the $1220 \mathrm{~cm}^{-1}$ mode) that were similar to those from the previous calculation.

(45) This was done by increasing the $\kappa$ value from 0.01 to 1 , where $\kappa$ is the line shape parameter given by $\Lambda / D$.

(46) The required adiabatic surfaces were calculated from diabatic surfaces and $H_{\mathrm{ab}}$ by utilitzing first-order perturbation theory as outlined by Sutin (Prog. Inorg. Chem. 1983, 30, 441). The overall scaling is achieved by ensuring that the ratio of $E_{\mathrm{op}}$ to twice $H_{\mathrm{ab}}$ equals the experimentally obtained value.

(47) A second complication is that wave packet evolution does not necessarily occur solely on the upper adiabatic surface, a problem discussed briefly in ref 11a and in considerably more detail by Zink and co-workers. ${ }^{46}$ Upon moving through the curve-crossing region, the wave packet (or a fraction of the wave packet) may jump to the lower diabatic surface and continue its evolution there (see Newton, M. D.; Sutin, N. Annu. Rev. Phys. Chem. 1984, 35, 437). An accurate calculation would need to follow the wave packet evolution and keep track of curve crossing, which for the high- frequency vibrations present in this system might involve several oscillations. In the absence of appreciable damping, upper-surface/lower-surface wave packet interference effects might also be needed to be considered.

(48) The need to propagate on adiabatic surfaces has been recognized previously. In fact, adiabatic surfaces have already been used by Zink and co-workers to calculate intervalence absorption bands for mixed-valence complexes (Simoni, E.; Reber, C.; Talaga, D.; Zink, J. I. J. Phys. Chem. 1993, 97, 12678. Zink, J. I.; Reber, C. Coord. Chem. Rev. 1991, 111, 1.). Adaptation and extension of their approach to include absolute scattering cross sections, several vibrational modes, detailed solvent reorganizationa effects, and detailed dephasing effects would almost certainly resolve the problems encountered here. Their analysis also elegantly handles wave packet curve-crossing and partial curve-crossing effects. Wave packet propagation on adiabatic surfaces (although not yet extended to the calculation of absorbance and Raman excitation profiles) has also been addressed by Coalson and co-workers. See, for example, the following. Coalson, R. D. J. Phys. Chem. 1996, 100, 7896.

(49) In an earlier report, ${ }^{5} H_{\mathrm{ab}}$ was estimated at about $1200 \mathrm{~cm}^{-1}$. The estimate was derived from the ratio of the IVCT oscillator strength to the charge-transfer distance, as outlined by Hush. ${ }^{3}$ Since we now believe that the effective charge-transfer distance is only about $70 \%$ of the value used previously, we have revised our $H_{\mathrm{ab}}$ estimate to $1700 \mathrm{~cm}^{-1}$.

(50) For a diatomic, $\Delta=(1 / 2) f(\Delta a)^{2}$, where $f$ is a force constant and $\Delta a$ is the change in bond length.

(51) Other parameters include a lifetime broadening of $100 \mathrm{~cm}^{-1}$ and a transition length of $0.84 \AA$.

(52) In addition, the displacement $\left(\Delta_{k}\right)$ between the minima on the adiabatic surfaces is less than the displacement between the minima on the diabatic surfaces (see Figure 3). However, this correction is comparatively small and has been ignored.

(53) For a similar analysis of an electrode reaction, see the following. Selmarten, D. C.; Hupp, J. T. J. Chem. Soc., Faraday Trans. 1996, 92 , 3909.

(54) $f(F C)$ is

$$
\begin{array}{r}
\exp \left[-\sum_{j} S_{\mathrm{j}} \operatorname{coth}\left(\frac{\hbar \omega_{j}}{2 k T}\right)\right] \prod_{j} \sum_{m_{j}=-\infty}^{\infty} \exp \left(\frac{m_{j} \hbar \omega_{j}}{2 k T}\right) I_{\mathrm{m}_{\mathrm{j}}}\left[S_{\mathrm{j}} \operatorname{csch}\left(\frac{\hbar \omega_{j}}{2 k T}\right)\right] \times \\
\exp \left[\frac{\left(\Delta E+\sum_{j} m_{j} \hbar \omega_{j}+\chi_{\mathrm{s}}\right)^{2}}{4 \chi_{\mathrm{s}} R T}\right]
\end{array}
$$

(55) (a) Brunschwig, B. S.; Sutin, N. Commun. Inorg. Chem. 1987, 6 , 209. (b) Spears, K. G. J. Phys. Chem. 1987, 88, 167. (c) Sarai, A.; Kakitani, T. Chem. Phys. Lett. 1981, 77, 427.

(56) For a similar analysis, see the following. Brunschwig, B. S.; Logan, J.; Newton, M. D.; Sutin, N. J. Am. Chem. Soc. 1980, 102, 5798

(57) Another motivation for introduction of the augmented quartic surface approach was the recognition by Nelsen et al. ${ }^{8}$ that harmonic surfaces yield symmetrical (Gaussian) IVCT absorption line shapes, provided that the pertinent vibrational frequencies are very small in comparison to $\chi_{\mathrm{s}}$. Quartic surfaces, on the other hand, can yield asymmetrical IVCT line shapes similar to those encountered experimentally. It should be noted, however, that purely harmonic surfaces can also lead to asymmetrical line shapes if large vibrational frequencies are employed.

(58) The averaged vibrational energy is determined using 9

$$
v_{v}=\left[\sum_{q}\left(\frac{v(q)^{2} \chi(q)}{\chi_{\text {vib,total }}}\right)\right]^{1 / 2}
$$

where the sum runs over the $q$ vibrational modes with frequencies $v(q)$ and reorganization energies $\chi(q)$. The value obtained here is $1053 \mathrm{~cm}^{-1}$.

(59) $\Delta \mathrm{G}^{*}$ was calculated from

$$
\Delta G^{*}=\frac{\left(\frac{\chi_{\mathrm{tot}}}{4}\right)\left(1+\frac{C}{4}\right)}{1+C}-H_{\mathrm{ab}}+\frac{H_{\mathrm{ab}}{ }^{2}}{\chi_{\mathrm{tot}}}
$$

where $C$ is the quartic factor, taken to be 0.2 . 